\title{
Upper respiratory tract infections: Hidden complications and management plan
}

\begin{abstract}
Upper Respiratory Tract Infection (URTI) or acute nasopharyngitis is the most common disease in pediatrics. However, it is not always simple and transient as its causative pathogens are changing and challenging. Local and Systemic Septic complications of bacterial URTI are common and systemic aseptic complications of streptococcal nasopharyngitis are not uncommon. However, there is a great debate about the management plan because of two facts: It is the most common cause of antibiotic use and misuse. ${ }^{1}$ and all septic and aseptic complications are actually preventable if bacterial URTI was properly treated with antibiotics. ${ }^{2}$. The aim of this article is to summarize facts about septic and aseptic complications of URTI and to illustrate the best management plan including wise use of antibiotics to save pediatric patients not only from overtreatment, but also from under treatment.
\end{abstract}

Keywords: upper respiratory tract infection, interfering flora, vaccine, streptococcus pyogenes group a, m- protein; toxic shock syndrome, scarlet fever, post streptococcal glomerulonephritis, rheumatic fever, pandas, monoclonal antibody, antibiotic
Volume 7 Issue I - 2017

\author{
Gihad I Alsaeed,' Ibrahim G Alsaeed, ${ }^{2}$ Tamer \\ $M$ Rizk $^{3}$ \\ 'Department of Pediatrics, Al-Takhassusi Hospital, Saudi Arabia \\ ${ }^{2}$ Fifth Year Medical Student, Humanitas University, Italy \\ ${ }^{3}$ Pediatric Neurology Department,Al-Takhassusi Hospital, Saudi \\ Arabia
}

Correspondence: Gihad I Alsaeed, MD, MSc, DCH (Ire), MRCPCH (UK), Department of Pediatrics, Al-Takhassusi Hospital, Dr. Sulaiman Al-Habib Medical Group, HMG Takhassusi Hospital, P.box: 2000, Riyadh, I I 393, Saudi Arabia,

Email gehadalsaeed@yahoo.com

Received: June 07, 2017 | Published: July 28, 2017
Abbreviations: URTI, upper respiratory tract infection; HSV, herpes simplex viruses; EBV, epstein bar virus; RSV, respiratory syncytial virus; STSS, streptococcal toxic shock syndrome; PT, prothrombin time; PTT, partial thromboplastin time; RADT, rapid antigen detection test; ASO, anti-streptolysin anti bodies; APSGN, acute post streptococcal glomerulonephritis; BUN, blood urea nitrogen; RHD, rheumatic heart disease; HAAT, high alanine amino transferase; ACEI, angiotensin converting enzyme inhibitor; ESR, erythrocyte sedimentation rate

\section{Introduction}

Acute Nasopharyngitis or (URTI) is inflammation of the nasal, paranasal, and pharyngeal mucosa. It usually diffuses in all tissues especially in infants and small children due to immaturity of local anatomical barriers. Viruses account for $65 \%$ of cases mainly Rhinovirus, Influenza, and Parainfluenza. Other viruses like Respiratory syncytial virus (RSV), Herpes simplex viruses (HSV), Adeno virus, Epstein bar virus (EBV) are possible causes. ${ }^{3}$ Bacteria account for $35 \%$ of cases; Streptococcus pyogenes (GAS) causes $25 \%$ of bacterial nasopharyngitis. Streptococcus pneumonia, Haemophilus influenza, Gonorrhea, Moraxella, and Mycoplasma contribute to the remaining $10 \% .^{3}$ Minority of nasopharyngitis may be caused by autoimmune systemic inflammation like SLE and Kawasaki disease. Or due to severe irritation by reflux (GERD), Alcohol, or smoking. Clinical presentation may be any combination of the following signs and symptoms: dryness, burning sensation of the throat above soft palate, pain and discomfort localized to the back of the nose with some difficulty in swallowing, fever, enlarged cervical lymph nodes, swollen mucosa, and whitish exudates might be found

\section{The interfering flora}

Normal nasopharyngeal Ecosystem contains aerobic flora like alpha hemolytic streptococci (Streptococcus Mitis and Streptococcus sanguis), and anaerobic bacteria like Prevotella Melaninogenica or Peptostreptococcus. ${ }^{4}$ Bactericidal protein "Bacteriocin" produced by those organisms helps to kill pathogens when they invade the respiratory mucosa. Unfortunately, many factors can destroy these interfering flora; viral infections, air conditioner effect, direct or indirect smoking, and contact with nasal droplets of smokers can decrease the colonization with interfering flora and increase colonization with pathogenic bacteria like Streptococcus Pneumonia, Staphylococcus Aureus, and Haemophilus influenza. ${ }^{5}$ Antibiotics used previously influence the composition of the nasopharyngeal flora. For example, second or third generation Cephalosporins do not eliminate the interfering flora as does amoxicillin. ${ }^{6}$ Air conditioner units eliminate humidity from closed buildings; at the same time coils and filters are good medium for atypical pathogens like Legionella, Listeria, and aspergillus species to grow and pollute air stream. The incidence of respiratory infections in air conditioned buildings community is 2.5 higher than those living in open environment. ${ }^{7}$ Fixing an Ultra Violet light source close to the filter and coils inside a given air conditioner seems effective to clear the unit from fungi and bacteria.?

\section{The individual type of pathogens}

The individual type of bacteria that causes bacterial URTI in a previously healthy child depends on:

a. Immunization history.

b. Previous antimicrobial therapy.

c. The type of glycoprotein receptors in respiratory epithelial cell membrane.

d. Presence of normal flora capable to prevent the growth of pathogens.

e. Viral infection: the nasopharynx is also the reservoir for pathogens capable of causing respiratory infection. During viral infection, potential pathogens can relocate into the sinus cavity, middle ear, or down to lungs and may cause active infection just by this relocation..$^{1,8}$

f. Seasonal and geographical variations. 
Streptococcus Pyogenes (group A, Beta hemolytic), or (GAS) Still the most important pathogen; Streptococcus Pyogenes can present molecules on its cell wall, like M-protein identical to molecules found on cell membrane of human tissues in the skin, heart, joints, and brain. This mechanism of fooling the immune system is called (Molecular Mimicry). GAS produces at least 18 exotoxins able to act as Super antigens that can avoid the MHC-II system processing immune response and induce exaggerated, nonspecific, harmful humoral response. All aseptic complications recognized till now following bacterial URTI are induced by GAS. ${ }^{9}$

\section{Septic complications}

Bacteremia or Systemic dissemination of bacteria and invasion of blood stream is not uncommon. Occult bacteremia usually causes fever without clear focus of infection. Most cases in infants were caused by Streptococcus Pneumonia, and 10\% of them developed serious illnesses. ${ }^{9,10}$ Direct spread of infection occurs more frequently in children than adults. Relocation of pathogens from the nasopharynx during viral infections may cause: Acute sinusitis, Otitis Media, Mastoidites, orbital cellulitis, periorbital cellulitis, facial cellulitis, Peritonsillar abscess, or Retropharyngeal Abscess.

\section{Aseptic complications of URTI}

\section{Streptococcal Toxic Shock Syndrome STSS}

It is a toxin induced immune mediated life-threatening illness precipitated by staphylococcus aureus or GAS colonization or infection. Persons of all ages affected, most do not have predisposing disease. ${ }^{11}$ Toxic shock syndrome toxin-1(TSST-1) or (Enterotoxin-B) produced by staph and Pyogenic Exotoxins A, B, C produced by GAS-M3 strains can act as super antigens causing massive release of deregulated cytokines that destroy tissues and causes shock. Pyrogenic exotoxins A\&B isolated in most cases in USA. TSST-1 Anti toxin specific antibodies were found in $90 \%$ of adults in USA, which seems protective against TSS. Absence of these antibodies is a risk factor. ${ }^{12}$

Diagnosis of streptococcal (STSS) needs the presence of hypotension (systolic blood pressure less than 5th centile for age, or $90 \mathrm{~mm}$ hg after 12 years old.) plus two of the following criteria:

a. Renal involvement: Elevated blood urea nitrogen (BUN), Creatinine (CR).

b. Liver involvement: Low Albumin, High Alanine AminoTransferase (ALT).

c. Coagulopathy: Thrombocytopenia, High Prothrombin Time (PT), and/or Partial Thromboplastin Time (PTT).

d. Acute respiratory distress syndrome

e. Necrotizing fasciitis, desquamative rash on palms and soles.

Diarrhea, vomiting, headache and non-focal neurologic abnormalities may be found. These criteria may accumulate over 4 days period and the rash after 2 weeks. Blood culture usually is negative. ${ }^{12}$ Streptococcal skin Infection at the site of minor trauma or varicella could be the primary infection. However, port of entry cannot be found in at least half the cases. Pharyngitis can be the primary infection. ${ }^{11,12}$

Treatment: Supportive, Clindamycin has the ability to eradicate GAS infection and suppress toxin synthesis. Vancomycin and other new anti-staph like: Tedizolide, Oritavancin, Dalbavancin can be used according to $\mathrm{C} / \mathrm{S}$ results. Keeping in mind that $20 \%$ of mild to moderate streptococcal infections are refractory to penicillin, penicillin is not recommended in such invasive situation as an empirical treatment. Monoclonal antibodies (mAb-4G3, mAb-5G2) improve prognosis considerably and seems promising effective therapy to naturalize toxins and autoimmune effects. ${ }^{12,13}$

\section{Scarlet fever}

Induced by streptococcal pyrogenic exotoxin A, usually within 1272 hours of GAS pharyngitis. The typical scarlet fever erythematous rash is worst in skin folds, usually spares perioral triangle, and often followed by exfoliative rash on digits. Straw berry tongue and cervical lymphadenitis in addition to the rash make it clinically similar to Kawasaki disease. Anti-streptolysin anti bodies (ASO) titer is usually not elevated. Rapid Antigen Detection Test (RADT) and/ or throat culture is usually positive. Treatment for 10 days with beta lactam antibiotic is enough even if the swollen tonsils and lymph nodes may take weeks to return to normal. ${ }^{14}$ Scarlet fever attack in Hong Kong in 2011 was resistant to macrolides but still sensitive to beta lactams. ${ }^{15}$ The most common associated complication of scarlet fever is Glomerulonephritis.

\section{Acute post streptococcal glomerulonephritis (APSGN)}

APSGN is an immune complex disease that follows $25 \%$ of GAS skin infections after 3-6 weeks, and 10\% of GAS nasopharyngitis after 1-2 weeks. Clinical presentation usually includes hematuria, proteinuria, edema, and hypertension. ${ }^{16}$ However, in $50 \%$ of cases there is only abnormal urine analysis and in rare cases hypertensive encephalopathy may be the presenting feature of APSGN. Complement C3 level is low while C4 is usually normal. ASO titer remains normal in skin infections while Anti-DNase B antibodies (anti-deoxy ribonuclease B) might be elevated. The relative long incubation period and the negative ASO after skin infections makes it difficult to prove GAS infection. Prognosis usually is good with resolution of hypertension and edema in 2 weeks, hematuria in 3-6 weeks, and proteinuria in 3-6 weeks. Microscopic Hematuria may persist 6 months. Complement C3 returns to normal in 8-10 weeks. Persistence of proteinuria and hematuria up to one year is not an indicator of poor renal function. While the outcome still very good in epidemic APSGN in the developing countries, sporadic cases in the developed countries may have poor prognosis with $33 \%$ of cases end with renal damage, episodic abnormal urine analysis (proteinuria and /or hematuria) might be found up to 20 years thereafter. ${ }^{16}$

\section{Rheumatic fever (RF)}

A systemic immune mediated multisystem inflammatory disease that occurs few weeks after a GAS throat infection, rarely with streptococcal infection of other sites. The structural similarity between many cardiac tissues and the streptococcal antigen M-Protein activates cytotoxic and humoral immune reaction that damages the heart tissue. Rheumatic heart disease (RHD) is the cardiac manifestations of RF and is associated with inflammation of the valves, myocardium, and/or pericardium. ${ }^{17,18}$ Around 3-5\% of untreated streptococcal pharyngitis turns into rheumatic fever, most patients are kids 5-15 years old. Rheumatic heart disease after one attack of RF is $30-70 \%$. However, $66 \%$ of RHD were diagnosed without a previous acute rheumatic fever diagnosis in the United States 2015.

A substantially large-scale study included 9800 asymptomatic school aged students using Echo Doppler in Riyadh Saudi Arabia, 2011 disclosed that the prevalence of RHD is 0.73 per thousand. $50 \%$ of them without previous history of RF. ${ }^{17}$ Another study found 
that RF continue to occur in Saudi Arabia despite the progress made in the socioeconomic development of the country and this is often associated with severe cardiac involvement in $53 \%$ of cases. ${ }^{19}$

Many patients with RHD present with shortness of breath only when they are 15 to 40 years old mostly because the clinical criteria of rheumatic fever may not occur at the same time and the Mitral valve regurgitation could be trivial and asymptomatic for years before the severe stenosis phase. While arthritis need 2-4 weeks to be seen, carditis needs 4-8 weeks, and chorea might be seen after 1-6 months from the onset of the primary GAS pharyngitis. ${ }^{17,18}$ It seems logic to remember that there is no cure for RF and no cure for rheumatic heart disease. Diagnosis of RF still a clinical decision depending on the criteria of Duckett-Jones; Major Criteria: Arthritis, carditis, subcutaneous nodules, erythema marginatum, Sydenham chorea. Minor Criteria: arthralgia, fever, high ESR, CRP. Prolonged PR interval. Two major or one major plus 2 minor criteria are needed for the diagnosis of rheumatic fever, and we need to confirm recent streptococcal infection with positive RADT, positive $\mathrm{C} / \mathrm{S}$ or high ASO. ASO alone has no value or correlation to the diagnosis of $\mathrm{RF}$ and has no value to follow up the course of disease or even to evaluate the response to treatment or worsening or relapse. It indicates nothing more than a recent GAS throat infection. Minor criteria alone are not diagnostic even if all are present. Similarly, if we consider carditis as the only major criteria available we cannot consider prolonged PR as the supporting minor criteria. ${ }^{18}$ Treatment of RF during acute attack may need Penicillin G Benzathine (1.2 million international units (MIU) $>27 \mathrm{~kg}$ or 0.6 MIU $<27 \mathrm{~kg}$. Oral amoxicillin, erythromycin or Cephalosporins for 10 days all are good alternatives. Aspirin and /or non-steroid antiinflammatory drugs for acute arthritis. Bed rest diuretics, angiotensin converting enzyme inhibitor (ACEI), with or without prednisone for acute carditis. Haloperidol, carbamazepine diazepam might be helpful to control chorea. Duration of treatment of the acute phase can be monitored by the level of erythrocyte sedimentation rate (ESR), and $\mathrm{C}$ reactive protein (CRP). ASO is not useful at all for this purpose. ${ }^{17,18}$

\section{Autoimmune neuropsychiatric disorder associated with streptococcal infection (PANS/PANDAS)}

Pediatric autoimmune neuropsychiatric disorders associated with streptococcal infections (PANDAS) are thought to happen due to cross-reaction to antibodies produced in response to group A streptococcal infections. Streptococcal Antigens Molecular Mimicry with different neurons induce production of antibodies that interfere with Basal Ganglia and link neurons function and may result in a variable combination of Tics, Obsessive Compulsive Disorder (OCD), Enuresis, anxiety, or school performance deterioration. Sudden onset of symptoms after GAS pharyngitis usually presents. Diagnosis criteria for PANDAS; Pre-pubertal onset, sudden or episodic onset of symptoms, Hyperactivity, choreiform, or adventitious movements, Tics and/or OCD and Evidence of previous streptococcal infection. ${ }^{20,21}$

Treatment: Selective Serotonin Reuptake Inhibitors (SSRI), intravenous immunoglobulin (IVIG), Behavioral therapy, Long term prophylactic antibiotics against GAS is still controversial, however prompt and early treatment for GAS pharyngitis is necessary to prevent exacerbation. Tonsillectomy failed to show clear benefit according to systemic review. Tonsillectomy should be considered according to current surgical guidelines. ${ }^{20-22}$ There is lack of high level, long term studies about the management plan for PANS/PANDAS and no guidelines/agreed recommendations to be followed. Behavioral reversal therapy will remain a considerably safe option when applied in selected patients. ${ }^{22}$

\section{Streptococcal rapid antigen detection test (RADT)}

To confirm streptococcal infection; this simple test can be done on nasopharyngeal swabs. It should be taken from both tonsils and the posterior nasopharyngeal mucosa away from the tongue. Its specificity is $95 \%$ but sensitivity is only $70 \%$. It is worth mentioning that $20 \%$ of asymptomatic individuals carry GAS in their nasopharynx. USA guidelines recommend antibiotic treatment in symptomatic patients with positive RADT. If it is negative then $\mathrm{C} / \mathrm{S}$ should be done. European guidelines relying on RADT only. 4 types of RADT test are available now, the best of them is the Optical Immunoassay with sensitivity $94 \%$ and specificity $99 \%{ }^{23}$

\section{Management plan for upper respiratory infection}

URTI is the most common cause of antibiotic use in pediatrics in spite of all the argument about antibiotic over use and abuse. Many criteria and scores were developed to help clinicians find their way for the best use of antibiotics in treating URTI.

However, it might be easier to put patients in 2 main groups:

1. Suspected viral URTI in younger than 5 years child with flu like symptoms, cough, rhinitis, conjunctivitis, and congested throat, without cervical lymphadenitis and short duration of symptoms about 5-7 days only. ${ }^{24}$.

2. Suspected bacterial URTI in 5 years or older child, with strawberry tongue, lymphadenitis, and whitish exudates on tonsils, petechial rash on soft palate, and erythematous skin rash most in folds. Other scenario for bacterial infection is any URTI case that persists 10 days or more even without fever.

Patients with suspected viral infection need no investigations and should not be given more than symptomatic treatment. Patients with suspected bacterial infection need to perform the RADT. If it is positive then antibiotic is recommended. However, if it is negative, $\mathrm{C} / \mathrm{S}$ is indicated. If both are negative antibiotic should be avoided. It is still controversial whether to start antibiotics for 3 or 4 days till the $\mathrm{C} / \mathrm{S}$ results are available. Guidelines of the American Family Physician advice to give antibiotic till the result of $\mathrm{C} / \mathrm{S}$ is available in all areas were Rheumatic fever is high risk, like tropical and developing countries, poor and crowded societies, poor accommodation and ventilation units. ${ }^{24}$

\section{Which Antibiotic to Use?}

Streptococcus pyogenes is not a lactamase producing bacteria. That is why amoxicillin still considered first line of treatment for bacterial nasopharyngitis and penicillin for isolated streptococcal tonsillitis. Cephalosporins 1st or 2nd generations can be used as alternative to the 1 st line or as a 2 nd line if the 1 st line failed. Macrolides can be used if patient is allergic to penicillin or cephalosporin. When bacterial tonsillitis is part of a diffuse URTI or when the causative bacteria Is not identified penicillin is not recommended.

Suggested antibiotic treatment.

a. Oral Penicillin V: $250 \mathrm{mg}$ BID/TID for 10 days.

b. Intramuscular Penicillin G Benzathine: 600000 units $(<27 \mathrm{~kg})$ $\& 1,200000$ units $(>27 \mathrm{~kg})$.

c. Amoxicillin: $40-90 \mathrm{mg} / \mathrm{kg} /$ day BID or TID for 10 days.

d. Azithromycin: $12 \mathrm{mg} / \mathrm{kg} /$ day OD for 5 days

e. Clarithromycin: $7.5 \mathrm{mg} / \mathrm{kg} /$ day BID for 10 days 
f. Erythromycin: $40 \mathrm{mg} / \mathrm{kg} /$ day TID or QID for 10 days.

g. Cephalexin: $12.5 \mathrm{mg} / \mathrm{kg}$ (up to $500 \mathrm{mg}$ per dose) BID or TID. MAX $1 \mathrm{gr} /$ day for 10 days.

h. Cefaclor: $20-40 \mathrm{mg} / \mathrm{kg} /$ day BID or TID (max $1 \mathrm{gm} /$ day) for 10 days.

\section{Decision making}

1. If RADT test is negative in clinically bacterial URTI is it suitable to give antibiotics? No.

2. If it seems a nonspecific URTI for 10 days or more is it suitable to give antibiotics? YES. Infants and small children usually do not demonstrate localized signs and symptoms, it is unusual for viral URTI to persist more than 7 days.

3. If it seems non-localized bacterial URTI is it suitable to give penicillin? No. There is a high rate of treatment failure using penicillin in such cases even if it is caused by streptococcus pyogenes.

4. Why beta lactam antibiotics may fail to treat GAS infection while it is not a lactamase producing pathogen? GAS is a unique bacterium it can harness other lactamase producing bacteria present in the throat to destroy beta lactam antibiotics and survive.

5. If it is a confirmed case of streptococcal URTI and after 3 days of oral penicillin still there is no clinical improvement, is it suitable to discontinue penicillin? YES. Within 48 to 72 hours there should be good clinical response. Even with GAS tonsillitis the failure rate of penicillin is $20 \%$.

6. If the case is streptococcal tonsillitis but after 10 days of oral treatment the $\mathrm{C} / \mathrm{S}$ of the throat still positive.is it suitable to continue treatment till $\mathrm{C} / \mathrm{S}$ is negative? No. it is not recommended to repeat $\mathrm{C} / \mathrm{S}$ after treatment, and not recommended to treat asymptomatic carriers.

7. If the RADT is negative in clinically bacterial URTI in area were the risk of RF is relatively high, is it suitable to give antibiotic till the $\mathrm{c} / \mathrm{s}$ result is available? YES.

8. If the RADT is negative in clinically bacterial URTI, C/S is negative after 3 days, but the child still febrile is it suitable to continue antibiotic? No. Viral infections like EBV can cause very similar scenario.

9. If it is a known case of recurrent streptococcal tonsillitis, are there better options than penicillin? Clindamycin and other macrolides have better eradication rate in such cases. Some experts recommend adding 5 days of oral Clindamycin to the 10 days course of beta lactam.

\section{Acknowledgments}

None.

\section{Conflicts of interest}

The authors declare no conflicts of interest.

\section{References}

1. Mayo Clinic Staff. Antibiotics misuse outs you and others at risk. 2014.

2. Brook I, Foote PA. Bacterial interference and beta-lactamase producing bacteria in the adenoids after antimicrobial therapy. Rev infect Dis. 1997;25:493.
3. Carrillo Marquez MA. Bacterial Pharyngitis: background, pathophysiology, epidemiology. 2016.

4. Brook I, Gober AE. Bacterial interference in the nasopharynx following antimicrobial therapy of acute otitis media. J Antimicrob Chemother. 1998;41(4):489-492.

5. Walls T, Power D, Tagg J. Bacteriocin-like inhibitory substance (BLIS) production by the normal flora of the nasopharynx:potential to protect against otitis media? J Med Microbiol. 2003;52 (Pt 9):829-833.

6. Varon E, Levy C, De La Rocque F, et al. Impact of Antimicrobial Therapy on Nasopharyngeal Carriage of Streptococcus pneumoniae, Haemophilus influenzae, and Branhamella catarrhalis in children with Respiratory Tract Infections. Clin Infect Dis. 2000;31(2):477-481.

7. Thompson C. Negative Health Effects of central Air conditioning. 2014.

8. Tsai YW, Huang WF, Wen YW, et al. The Relationship between Influenza Vaccination and Outpatient Visits for Upper Respiratory infection by the Elderly in Taiwan. International Society for Pharmacoeconomics and Outcomes Research (ISPOR). 2007;10(2):117-127.

9. Kirvan CA, Swedo SE, Kurahara D, et al. Streptococcal mimicry and antibody-mediated cell signaling in the pathogenesis of Sydenham's chorea. Autoimmunity. 2006;39(1):21-29.

10. Weinberg GA. Occult Bacteremia and Fever without Apparent Source in Infants and Young Children. 2015.

11. Stevens DL. Streptococcal toxic-shock syndrome:spectrum of disease, pathogenesis, and new concepts in treatment. Emerg Infect Dis. 1995;1(3):69-78.

12. Stevens DL. Treatment of streptococcal toxic shock syndrome. 2007.

13. Aguilar JL, Varshney AK, Pechuan X, et al. Monoclonal Antibodies protect from Staphylococcal Enterotoxin K (SEK) induced toxic shock and sepsis by USA 300 Staphylcoccus aureus. J Virulence. 2016;1(3).

14. Scotoodian B. Scarlet Fever. Medscape. 2016.

15. Luk EYY, Lo JYC, Amy ZL Li, et al. Scarlet fever Epidemic, Hong Kong. Emerg Infect Dis. 2012;18(10):1658-1661.

16. Niaudet P. Poststreptococcal glomerulonephritis. 2016.

17. Qurashi MA. The pattern of acute rheumatic fever in children:Experience at the children's hospital, Riyadh, Saudi Arabia. J Saudi Heart Assoc. 2009;21(4):215-220.

18. Beaudoin A, Edison L, Camille E Introcas, et al. Acute Rheumatic Fever and Rheumatic Heart Disease Among Children-American Samoa, 20112012. Centers for Disease Control and Prevention (CDC) Morbidity and Mortality Weekly Repost (MMWR). 2015;64(20):555-558.

19. Ahmed M. Rheumatic Heart Disease. 2015.

20. Murphy TK, Kurlan R, Leckman J. The immunobiology of Tourette's disorder, pediatric autoimmune neuropsychiatric disorders associated with Streptococcus, and related disorders:a way forward. J Child Adolesc Psychopharmacol. 2010;20(4):317-331.

21. Williams KA, Swedo SE, Farmer CA, et al. Randomized, Controlled Trial of Intravenous Immunoglobulin for Pediatric Autoimmune Neuropsychiatric Disorders Associated with Streptococcal Infections. $J$ Am Acad Child Adolesc Psychiatry. 2016;55(10):860-867.

22. Farhood Z, Ong AA, Discolo CM. PANDAS: A systematic review of treatment options. Int J Pediatr Otorhinolaryngol. 2016;89:149-53.

23. Gerber MA, Tanz RR, Kabat W, et al. Optical immunoassay tests for group A beta- hemolytic streptococcal pharyngitis. JAMA. 1997;277(11):899903.

24. Wong DM, Blumberg DA, Lowe LG. Guidelines for the use of antibiotics in Acute Upper Respiratory tract infections. Am Fam Physician. 2006;74(6):956-966. 